\title{
AOR
}

Selected Papers of \#AolR2021:

The 22nd Annual Conference of the

Association of Internet Researchers

Virtual Event / 13-16 Oct 2021

\section{FROM DIGITAL INFLUENCERS TO INFLUENCER-LABORATORIES. A CASE STUDY OF LIL MIQUELA}

\author{
Elias Bitencourt \\ Universidade do Estado da Bahia \\ Universidade Federal da Bahia \\ Grégori Castelhano \\ Universidade Federal da Bahia \\ Catarina Lopes \\ Universidade Federal da Bahia

\section{Introduction}

Digital influencers stand out as a phenomenon that mixes independent entrepreneurship and a profound interdependence with platform services. Treated as microcelebrities (Senft, 2008) that have achieved their status by attracting a large audience in social networks, digital influencers are frequently associated with people who use social capital acquired online to gain access to financial resources (Abidin, 2015). The literature tends to differentiate between digital influencers and celebrities in the traditional media based on two general factors: authenticity and entrepreneurship (Duffy \& Hund, 2015; Marwick, 2015, 2018)

However, if they are to appear authentic or real or to be financially successful on social networks, digital influencers must constantly play the visibility game (Cotter, 2019) with platform affordances to achieve online influence (Gerlitz \& Rieder, 2018).

This research focuses on an emerging influencer category: CGI influencers, or virtual influencers (Marwick, 2018). CGI influencers are characters created with computer modeling techniques that have profiles on social networks and sociomaterial trajectories built with the aid of digital marketing, business intelligence and media companies.

Most notable among the various successful examples is @lilmiquela. Created in 2016, Miquela presents herself as a pop artist. With more than 3 million followers on Instagram, 7000 subscribers on YouTube and 2.9 million on TikTok, she was

Suggested Citation (APA): Bitencourt, E; Castelhano, G; Lopes, C. (2021, October).From digital influencers to influencer-laboratories. A case study of Lil Miquela. Paper presented at AoIR 2021: The 22nd Annual Conference of the Association of Internet Researchers. Virtual Event: AolR. Retrieved from http://spir.aoir.org. 
considered one of the 25 most important internet personalities in 2018 by TIME${ }^{1}$. Behind this independent artist is Brud ${ }^{2}$, a Californian company founded in 2014 that specializes in robotics, $\mathrm{Al}$ and storytelling ${ }^{3}$ and has received more than 6 million dollars financial support from investors in the technology sector since $2017^{4}$.

The aim of this study was to map the ways in which Miquela acts on Instagram and to find traces that allow us to explore better the interrelationship between the Brud strategies and the appropriations of Instagram affordances that model the ways in which the CGI acts both as a fictional character and as a digital influencer. The main questions we asked were: (a) what Instagram affordances does Miquela prioritize in her actions in the feed? (b) how do Miquela and Brud appropriate these platform affordances to build a fictional world and the attributes of the CGI character? and (c) how does the intertwining of the appropriations of affordances and the storytelling approach impact the promotion of the character and the brands that she advertises in her feed?

We argue that Miquela represents a type of digital influencer for which the influencer itself acts as a laboratory used to investigate which sociomaterial arrangements create conditions that favor the production of online influence on digital platforms.

\section{Methodology}

We used Instaloader ${ }^{5}$ to extract 1089 posts available in Miquela's feed up to June 2021. We classified the posts by the type of affordance used (i.e., hashtags, location, profile mentions, profile tagging, type of media) and calculated post engagement based on the number of followers each year ${ }^{6}$. We found that the profile mention and tagging tools were the ones most used by Miquela, so we explored these in a bipartite network (posts/tags + mentions) in Gephi and found clusters characterized mainly by fashion, celebrity, luxury brand and Brud product profiles. We used these preliminary findings as guidance for the content analysis. The database was imported into ATLAS.ti and analyzed according to three criteria: (a) type of content promoted; (b) type of profiles tagged, cited or mentioned and (c) subjects used as context for the posts (topics). Finally, we filtered the posts with good engagement $(\mathrm{N}=390)$ and investigated cooccurrences among the categories adopted.

\footnotetext{
${ }^{1}$ https://time.com/5324130/most-influential-internet/

${ }^{2}$ http://brud.fyi/

${ }^{3}$ https://www.linkedin.com/company/brudinc

${ }^{4}$ https://www.crunchbase.com/organization/brud

${ }^{5}$ https://instaloader.github.io

${ }^{6}$ To recover the follower base before 2021, we used technical reports and news articles covering Miquela's career from 2016 onwards.
} 


\section{Findings and Discussion}
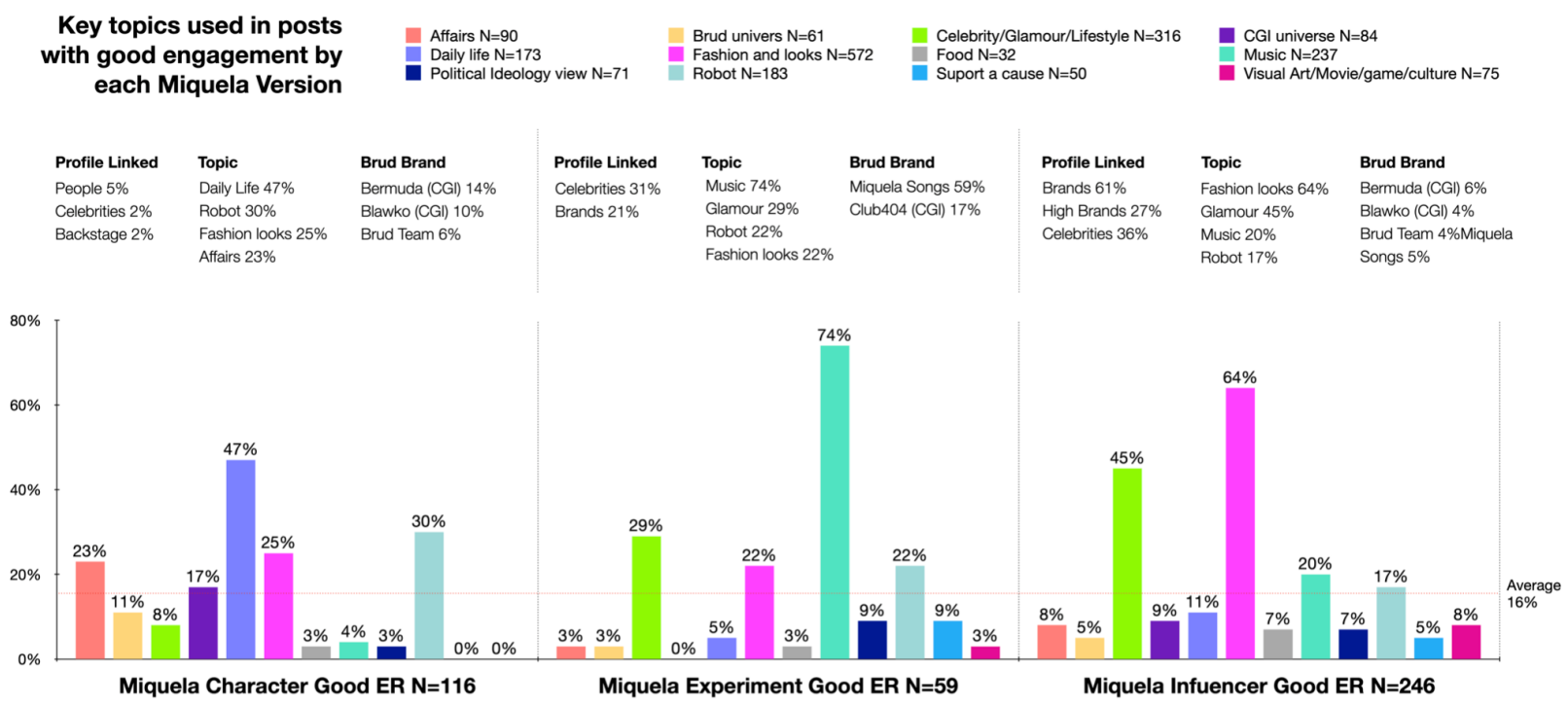

Figure 1. Summary of the different practices adopted by each version of Miquela. Source: the authors.

We observed three ways of acting (practices) that are characteristic of Miquela and operate interdependently. We called these sets of practices versions, namely: (a) Miquela the Fictional Character, (b) Miquela the Experiment and (c) Miquela the Influencer (Fig. 1).

We found that Miquela the Character adopts practices that appear to be aimed at testing subjects that will make up the central arcs of the fictional CGI narrative and that can be explored in the other two versions. In this version, the tagging and mention tools are used as a way of anchoring the CGI character in the real world (tagging and mentioning real partners and places) and as the company's digital signature.

In the experimental version, we observed that the use of tagging and mentioning features is more geared toward qualifying and promoting Miquela's own brand products (i.e., songs and merch $^{7}$ ) by targeting celebrities and deluxe brands in posts about musical topics. This version appears to aim to explore ways of reaching fashion and lifestyle niches in a controlled manner (using Miquela's products as experimental bait).

In the influencer version, we observed practices that focused more on brand content promotion with more directed use of tagging and mentioning tools; this version appears to combine the best results of the experimental version, the subjects that stand out most in the character and experimental versions and a greater number of brand profile tags.

Added to the fact that Brud raised substantial investment funding in the last few years, these findings could suggest that the three versions of Miquela act as an experimental

${ }^{7}$ https://bit.ly/3yg61gO 
model of what we here call "influencer-laboratories". Together, the three versions of Miquela mix the mimicry of "realism", "authenticity" (character version) and "entrepreneurship" (experimental and influencer versions) that characterize "influencers" (Abidin, 2015; Duffy, 2017), the fictional storytelling approach and the appropriation of platform affordances in a "controlled experiment". Such an experiment allows one to test and implement fine adjustments to both the fictional narrative of CGI and the practices that characterize Miquela as a digital influencer.

Although still only partial, these findings suggest that Brud may is promoting Miquela as a methodological experiment that allows one (a) to map which Instagram actions, topics and affordances exert more influence for each niche, (b) to refine these actions based on specific audience characteristics and (c) to design digital influencers and segmentedon-demand influence strategies.

Miquela illustrates the concept of influencer-laboratories and shows that when influence regimes are understood as a product that is interdependent on Instagram affordances, algorithmic recommendation systems and the platform political and economic model, the influencer him or herself may not act merely as an independent content creator or a microcelebrity whose career depends on the platform, but also as methodological bait for companies to learn how relationships of influence are built from the action of these characters on the internet.

\section{References}

Abidin, C. (2015). Communicative intimacies: Influencers and Perceived Interconnectedness. Ada: A Journal of Gender, New Media, and Technology, 8, 1-16.

Cotter, K. (2019). Playing the visibility game: How digital influencers and algorithms negotiate influence on Instagram. New Media \& Society, 21(4), 895-913. https://doi.org/10.1177/1461444818815684

Duffy, B.E; Hund, E. (2015) "Having it all" on social media: entrepreneurial femininity and self- branding among fashion bloggers. Social Media + Society 1(2): 1-11.

Gerlitz, C., \& Rieder, B. (2018). Tweets are not created equal: Investigating Twitter's client ecosystem. International Journal of Communication, 12, 528-547.

Marwick, A. (2015) Instafame: luxury selfies in the attention economy. Public Culture 27(75): 137-160.

Marwick, A (2018). The Algorithmic Celebrity: The Future of Internet Fame and Microcelebrity Studies: The Future of Internet Fame and Microcelebrity Studies. https://doi.org/10.1108/978178756749820181015

Senft, T. M (2008) Camgirls (Digital Formations), vol. 4. New York: Peter Lang. 